\title{
Iterative Methods for the Numerical Solution of Laplace's Equation on Non-Simply Connected Domain in $\mathbb{R}^{2}$
}

J.D. FLORES ${ }^{1}$, Department of Mathematical Sciences, The University of South Dakota, Vermillion, SD 57069-2390, USA

J. SAAVEDRA ${ }^{2}$, Department of Mathematics, Palomar College, San Marcos, CA 92069, USA.

\begin{abstract}
We consider the interior Dirichlet problem for Laplace's equation on non-simply connected two-dimensional regions with smooth boundaries. The approximate double layer density function is found by solving a system of Fredholm integral equations of second kind. Because of the non-uniqueness of the solution of the system we solve it using a technique based on the solution of the "Modified Dirichlet problem." The Nyström's method coupled with the trapezoidal rule is used as numerical integration scheme. The linear system derived from the discretization of the functional equation is solved using iterative Krylov conjugate gradient type methods. Theoretical and computational details of the method are presented.
\end{abstract}

\section{Introduction}

This paper describes a numerical method for the solution of the interior Dirichlet problem for Laplace's equation in multiply-connected regions bounded by smooth boundaries. The solution $u$ is represented as a double layer potential, and the resulting integral equation is then reformulated as a system of integral equations, each of them over the interval $[0,2 \pi]$. For the numerical solution, the Nyström's method is used with the trapezoidal rule as the numerical integration scheme.

The theory of integral equations and the existence of solution in a non-simply connected region has been studied in details. For example, see [4], [6], [8] and [9]. Numerical methods for solving Laplace's equations has been developed using integral equations, [2] and [7] among others.

The reformulation of the interior Dirichlet problem for a non-simply connected region is based on the theory developed by Mikhlin [8] and Muskhelishvili [9]. This uses the solution of the modified Dirichlet problem, to avoid the inconvenience produced by the non uniqueness of the solution of the system of equations of the original problem.

\footnotetext{
1 e-mail:jflores@usd.edu

${ }^{2}$ e-mail:jsaavedra@palomar.edu
} 


\section{Preliminaries}

Let $\Omega$ be a closed bounded non-simply connected subset of $\mathbb{R}^{2}$ with boundary $L=$ $\cup_{i=0}^{p} L_{i}, p \geq 1$ with $\left.\left.i\right) L_{i} \cap L_{j}=\Phi, i \neq j, i i\right) L_{i}$ is a simple smooth contour, all $i=0,1, \ldots, p$, iii) $L_{0}$ contains the other curves within its interior.

The Dirichlet problem is:

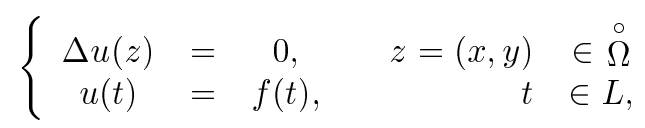

where $f$ is a real continuous function on $L$.

As it was suggested by Muskhelishvili [9] the solution of the Dirichlet problem can be obtained as a real part of a holomorphic function on $\stackrel{\circ}{\Omega}$ by transforming the original problem into the modified Dirichlet problem

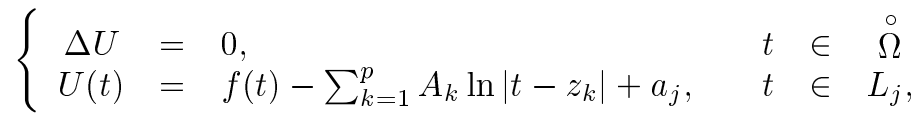

$j=0,1, \ldots, p, a_{0}=0, A_{k}$ are constants to be determined and $z_{k}$ is a point inside the region bounded by $L_{k}$. The equation (2.2) always has solution for a suitable choice of $\left\{a_{j}\right\}$.

The solution of (2.2) can be thought of as the real part of a Cauchy-type integral, and it involves a term of the form of the double layer potential

$$
\frac{1}{\pi} \int_{L} \mu(t) \frac{\partial \ln r}{\partial n} d s
$$

where $r=|t-z|$ and the direction of $\vec{r}$ is from $t$ to $z$. The element $d s$ belongs to the arc of the contour, $\frac{\partial}{\partial n}$ denotes the normal derivative in the direction of the outer normal vector $\vec{n}$, and $\mu(t)$ is called the double layer density function.

By letting $z$ tend to a point $\tau$ on $L$ we obtain an equation of the form

$$
\mu(\tau)+\frac{1}{\pi} \int_{L} \mu(t) \frac{\partial \ln r}{\partial n} d s=f(\tau)-\sum_{k=1}^{p} A_{k} \ln \left|\tau-z_{k}\right|+a(\tau), \tau \in L,
$$

with unknown function $\mu(t), t=t(s)$, and $a(t)=a_{j}, t \in L$.

We reduce the solution of the Modified Dirichlet problem to the solution of the integral equation

$$
\mu(\tau)+\int_{L}\left[\frac{1}{\pi} \frac{\partial \ln r}{\partial n} d s-S(\tau, t)\right] \mu(t) d s+\sum_{k=1}^{p} A_{k} \ln \left|\tau-z_{k}\right|=f(\tau),
$$

where the function

$$
S(\tau, t)=\left\{\begin{array}{l}
1 \quad \text { if }(\tau, t) \in L_{j}, j=1, \ldots, p \\
0 \quad \text { otherwise }
\end{array}\right.
$$


and so $\int_{L_{j}} S(\tau, t) d s \neq 0, \tau \in L_{j}, j=1, \ldots, p$, see [8, p. 170].

It turns out that this condition is necessary for the unique solvability of (2.4). In this way the $\int_{L_{j}} S(\tau, t) d s$ will have the values $c_{j}=\int_{L_{j}} \mu(t) d s, j=1, \ldots, p$ and $c_{0}=0$.

It is known that the homogeneous equation associated with (2.4) has no solution different from 0; see Muskhelishvili [9, p. 171]. The solution $\mu(t)$ of $(2.4)$ will also give a solution of (2.3) with $a_{j}=c_{j}=\int_{L_{j}} \mu(t) d s=0, j=1, \ldots, p$.

\section{The Functional Integral Operator}

We consider the solution of (2.4) as a function $\mu$ of the space $X=\left(\prod_{j=0}^{p} C\left(L_{j}\right)\right) \times$ $\mathcal{F}^{p},(\mathcal{F}=\mathbb{R}$ or $\mathbb{C})$. We denote $\mu=\left(\mu_{0}, \mu_{1}, \ldots \mu_{p}, A_{1}, \ldots, A_{p}\right)$ and we define the norm of $\mu$ by $\|\mu\|_{X}=\operatorname{Max}\left\{\left\|\mu_{i}\right\|_{C\left(L_{i}\right)},\left|A_{j}\right|, i=0,1, \ldots, p ; j=1,2, \ldots, p\right\}$. Clearly the space $X$ with this norm is a Banach space. Define the operator $\mathcal{T}$ on $X$ by the $(2 \mathrm{p}+1)$-tuple

$$
\mathcal{T} \mu=\left(\left\{\mu_{j}+\sum_{i=0}^{p} \mathcal{K}^{i j} \mu_{i}+\sum_{k=1}^{p} A_{k} \ln \left|(\cdot)-z_{k}\right|\right\}_{j=0}^{p},\left\{\int_{L_{j}} \mu_{j} d s\right\}_{j=1}^{p}\right)
$$

where $z_{k}$ is a point inside of the region bounded by $L_{k}$ and $\mathcal{K}^{i j}: C\left(L_{j}\right) \rightarrow C\left(L_{i}\right)$ is the integral operator defined by

$$
\mu_{j} \rightarrow \mathcal{K}^{i j} \mu_{j}(\tau)=\int_{L_{j}}\left[\frac{1}{\pi} \frac{\partial \ln r}{\partial n}-S(\tau, t)\right] \mu_{j}(t) d s .
$$

Lemma 1. The operator $\mathcal{T}$ is a Fredholm operator.

Proof. For a detailed proof see Saavedra[12]

Define the operator $\mathcal{K}: X \rightarrow X$

$$
\mathcal{K}(\mu)=\left(\left\{\sum_{i=0}^{p} \mathcal{K}^{i j} \mu_{i}+\sum_{k=1}^{p} A_{k} \ln \left|(\cdot)-z_{k}\right|\right\}_{j=0}^{p},\left\{\int_{L_{j}} \mu_{j} d s-A_{j}\right\}_{j=1}^{p}\right)
$$

and write $\mathcal{T}=I+\mathcal{K}$. In this form the solution of the modified Dirichlet problem (2.2) is equivalent to the solution of the following integral equation

$$
(I+\mathcal{K}) \mu=f
$$

where $f=\left(f_{0}, f_{1}, \ldots, f_{p}, 0, \ldots, 0\right)$, and the zeros are repeated $p$ times. Equation (3.3) is equivalent to equation (2.4), with the additional condition $\int_{L_{j}} \mu_{j} d s=0, j=$ $1, . ., p$. The integral equation (3.3) represents a system of integral equations formed 
by $2 p+1$ equations and $2 p+1$ unknowns functions and constants. It can be represented in matrix form by

$$
\left[\begin{array}{ccccccc}
I+\mathcal{K}^{00} & \mathcal{K}^{01} & \cdots & K^{0 p} & \ln \left|(\cdot)-z_{1}\right| & \cdots & \ln \left|(\cdot)-z_{p}\right| \\
\mathcal{K}^{10} & I+\mathcal{K}^{11} & \cdots & \mathcal{K}^{1 p} & \ln \left|(\cdot)-z_{1}\right| & \cdots & \ln \left|(\cdot)-z_{p}\right| \\
\vdots & \vdots & \ddots & \vdots & \vdots & \ddots & \vdots \\
\mathcal{K}^{p 0} & \mathcal{K}^{p 1} & \cdots & I+\mathcal{K}^{p p} & \ln \left|(\cdot)-z_{p}\right| & \cdots & \ln \left|(\cdot)-z_{p}\right| \\
0 & \int_{L_{1}}(\cdot) d s & \cdots & 0 & 0 & \cdots & 0 \\
\vdots & \vdots & \ddots & \vdots & \vdots & \ddots & \vdots \\
0 & 0 & \cdots & \int_{L_{p}}(\cdot) d s & 0 & \cdots & 0
\end{array}\right]\left[\begin{array}{c}
\mu_{0} \\
\mu_{1} \\
\vdots \\
\mu_{p} \\
A_{1} \\
\vdots \\
A_{p}
\end{array}\right]=\left[\begin{array}{c}
f_{0} \\
f_{1} \\
\vdots \\
f_{p} \\
0 \\
\vdots \\
0
\end{array}\right] .
$$

\section{The Numerical Scheme}

Assume that $L$ satisfies the same condition as before and that it is already parametrized. Define the operator $\mathcal{K}$, in (3.2), on the space $H=\left(\prod_{i=0}^{p} C[0,2 \pi]_{i}\right) \times \mathbb{R}^{p}$. To avoid overly complicated notation, we consider $p=1 . \quad \mathcal{K}$ is defined as

$$
\begin{aligned}
& \mathcal{K}: C[0,2 \pi] \times C[0,2 \pi] \times \mathbb{R} \rightarrow C[0,2 \pi] \times C[0,2 \pi] \times \mathbb{R} \\
& \mathcal{K}\left(\mu_{0}, \mu_{1}, A_{1}\right)=\left(\mathcal{K}^{00} \mu_{0}+\mathcal{K}^{01} \mu_{1}+A_{1} \ln \left|(\cdot)-z_{1}\right|,\right. \\
& \mathcal{K}^{10} \mu_{0}+\mathcal{K}^{11} \mu_{1}+A_{1} \ln \left|(\cdot)-z_{1}\right| \\
&\left.\int_{L_{1}} \mu_{1} d s-A_{1}\right)
\end{aligned}
$$

where $z_{1}$ is a point inside the region bounded by $L_{1}$. The integral equation has the form

$$
(I+\mathcal{K}) \mu=f
$$

This equation has a unique solution $\mu$. To solve (4.2) we choose the Nyström algorithm based on a convergent numerical integration scheme for each of the intervals. Because the smoothness assumption given for the boundary of $\Omega$, we take the same numerical scheme on each interval.

We consider two partitions for the interval $[0,2 \pi]$ of the form: $0=t_{q, 0}<t_{q, 1}<$ $\ldots<t_{q, n_{q}}=2 \pi, q=0,1, n_{0}$ and $n_{1}$ two natural numbers. Then we have

$$
\sum_{j=0}^{n_{q}} w_{j, n_{q}}^{r q} K^{r q}\left(s, t_{q, j}\right) \mu_{q}\left(t_{q, j}\right)=\int_{0}^{2 \pi} K^{r q}(s, t) \mu_{q}(t) d t+R_{n_{q}}\left(K^{r q} \mu_{q}\right) \quad q, r=0,1 .
$$

and,

$$
\sum_{j=0}^{n_{1}} w_{j, n_{1}} \mu_{1}\left(t_{1, j}\right)=\int_{0}^{2 \pi} \mu_{1}(t) d t+R_{n_{1}}\left(\mu_{1}\right)
$$


We also assume that for all $\mu \in H, \lim _{n_{q} \rightarrow \infty} R_{n_{q}}^{r q}=0 \quad q, r=0,1$ and $\lim _{n_{1} \rightarrow \infty} R_{n_{1}}\left(\mu_{1}\right)=0$. By the uniformly boundedness principle we have that

$$
\sup _{m} \sum_{j=1}^{m}\left|w_{j, m}^{r q}\right|<\infty, r, q=0,1 \quad \text { and } \quad \sup _{m} \sum_{j=1}^{m}\left|w_{j, m}\right|<\infty .
$$

Having determined the quadratures, we define the corresponding discrete operator. Denote the double index $k=(m, n)$ with $m, n, \in \mathbb{N}$, then

$$
\begin{aligned}
& \mathcal{K}_{k} \mu\left(s_{0}, s_{1}, A_{1}\right)= \\
& \left(\sum_{j=0}^{m} w_{j, m}^{00} \mathcal{K}^{00}\left(s_{0}, t_{0, j}\right) \mu_{0}\left(t_{0, j}\right)+\sum_{j=0}^{n} w_{j, n}^{01} \mathcal{K}^{01}\left(s_{0}, t_{1, j}\right) \mu_{1}\left(t_{1, j}\right)+A_{1} \ln \left|u_{0}-z_{1}\right|,\right. \\
& \sum_{j=0}^{m} w_{j, m}^{10} \mathcal{K}^{10}\left(s_{1}, t_{0, j}\right) \mu_{0}\left(t_{0, j}\right)+\sum_{j=0}^{n} w_{j, n}^{11} \mathcal{K}^{11}\left(s_{1}, t_{1, j}\right) \mu_{1}\left(t_{1, j}\right)+A_{1} \ln \left|u_{1}-z_{1}\right|, \\
& \left.\sum_{j=0}^{n} w_{j, n} \mu_{1}\left(t_{1, j}\right)-A_{1}\right)
\end{aligned}
$$

where the parametrization $f_{0}$ and $f_{1}$ of $L_{0}$ and $L_{1}$, respectively, are assumed and $u_{0}=f_{0}\left(s_{0}\right), u_{1}=f_{1}\left(s_{1}\right), \mu\left(s_{0}, s_{1}, A_{1}\right)=\left(\mu_{0}\left(s_{0}\right), \mu_{1}\left(s_{1}, A_{1}\right)\right)$. We observe that for every $k, \mathcal{K}_{k}$ is a compact operator.

Lemma 2. $\mathcal{K}_{k} \mu \rightarrow \mathcal{K} \mu$ as $k \rightarrow \infty$, i.e. as $m, n \rightarrow \infty$.

Proof. Define

$$
\begin{aligned}
& \mathcal{K}_{m}^{i 0} \mu_{0}\left(s_{i}\right)=\sum_{j=0}^{m} w_{j, m}^{i 0} K^{i 0}\left(s_{i}, t_{0, j}\right) \mu_{0}\left(t_{0, j}\right), \quad i=0,1 \\
& \mathcal{K}_{m}^{i 1} \mu_{1}\left(s_{i}\right)=\sum_{j=0}^{n} w_{j, n}^{i 1} K^{i 1}\left(s_{i}, t_{1, j}\right) \mu_{1}\left(t_{1, j}\right), \quad i=0,1
\end{aligned}
$$

Then

$$
\lim _{m \rightarrow \infty} \mathcal{K}_{m}^{i 0} \mu_{0}=\mathcal{K}^{i 0} \mu_{0}, \text { and } \lim _{n \rightarrow \infty} \mathcal{K}_{n}^{i 1} \mu_{1}=\mathcal{K}^{i 1} \mu_{1} \quad \mu_{0}, \mu_{1} \in C[0,2 \pi], i=0,1 .
$$

In addition, if $\mathcal{L}_{n} \mu_{1}(t)=\sum_{j=0}^{n} w_{j, n} \mu_{1}\left(t_{1, j}\right)$, then

$$
\mathcal{L}_{n} \mu_{1} \rightarrow \mathcal{L} \mu_{1}=\int_{L_{1}} \mu_{1} d s, \quad \mu_{1} \in C[0,2 \pi] .
$$

Thus, given $\epsilon>0$, there exists a natural number $N$ such tat for all $m, n \geq N$

$\left\|\mathcal{K}_{m}^{i 0} \mu_{0}-\mathcal{K}^{i 0} \mu_{0}\right\|_{C[0,2 \pi]}<\epsilon / 2$ and $\left\|\mathcal{K}_{m}^{i 1} \mu_{1}-\mathcal{K}^{i 1} \mu_{1}\right\|_{C[0,2 \pi]}<\epsilon / 2$ for $i=0,1$, and $\left|\mathcal{L}_{n} \mu_{1}-\mathcal{L} \mu_{1}\right|<\epsilon$.

Therefore 


$$
\begin{gathered}
\left\|\mathcal{K}_{k} \mu-\mathcal{K} \mu\right\|_{H}=\operatorname{Max}\left\{\left\|\mathcal{K}_{m}^{00} \mu_{0}-\mathcal{K}^{00} \mu_{0}+\mathcal{K}_{n}^{01} \mu_{1}-\mathcal{K}^{01} \mu_{1}\right\|_{C[0,2 \pi]},\right. \\
\left.\left\|\mathcal{K}_{m}^{10} \mu_{0}-\mathcal{K}^{10} \mu_{0}+\mathcal{K}_{n}^{11} \mu_{1}-\mathcal{K}^{11} \mu_{1}\right\|_{C[0,2 \pi]},\left|\mathcal{L}_{n} \mu_{1}-\mathcal{L} \mu_{1}\right|\right\}<\epsilon
\end{gathered}
$$

We now consider, the sequence of operators $\left\{\mathcal{K}_{k}\right\}, k \in \mathbb{N} \times \mathbb{N}$. It is known that this sequence of approximating operators need not to converge to the operator $\mathcal{K}$. But for the error analysis, it is sufficient to have

$$
\lim _{k \rightarrow \infty}\left\|\left(\mathcal{K}_{k}-\mathcal{K}\right) \mathcal{K}_{k}\right\|=0
$$

Lemma 3. The sequence of operators $\mathcal{K}_{k}: H \rightarrow H, k=(n, m)$ withn, $m \geq 1$, is collectively compact, i.e. the set

$$
B=\left\{\mathcal{K}_{k} \mu:\|\mu\|_{H} \leq 1 ; n, m \geq 1\right\}
$$

is precompact in $H$.

Proof. See Saavedra [12]. The proof is based on the theory of collectively compact operator approximation due to Anselone [1].

This result and the results from Anselone [1] imply (4.4), and this suffices for proving stability of the numerical method. The result (4.4) and the fact that $\left\{\mathcal{K}_{k}\right\}$ is uniformly bounded gives us

$$
\left\|\left(I+\mathcal{K}_{k}\right)^{-1}\right\| \leq \frac{1+\left\|(I+\mathcal{K})^{-1}\right\|\|\mathcal{K}\|}{1-\left\|(I+\mathcal{K})^{-1}\right\|\left\|\left(\mathcal{K}_{k}-\mathcal{K}\right) \mathcal{K}_{k}\right\|}<c
$$

where $c$ is a constant.

Theorem 1. Assume $\mathcal{K}^{i j} \in C\left(L_{i} \times L_{j}\right), i, j=0,1$, and $(I+\mathcal{K})^{-1}$ exist. Then for $m, n$ sufficiently large, the solution $\mu_{k}$ of $\left(I+\mathcal{K}_{k}\right) \mu_{k}=f$ and the solution $\mu$ of $(I+\mathcal{K}) \mu=f$ satisfy

$\left\|\mu_{k}-\mu\right\| \leq c \operatorname{Max}_{\alpha=0,1}\left\{\begin{array}{c}\operatorname{Max} \\ s \in L_{\alpha}\end{array}\left|R_{m}\left(\mathcal{K}^{\alpha 0} \mu_{0}\right)\right|+\underset{s \in L_{\alpha}}{\operatorname{Max}}\left|R_{n}\left(\mathcal{K}^{\alpha 1} \mu_{1}\right)\right|,\left|R_{n}\left(\mu_{1}\right)\right|\right\}$,

where $c$ is a constant and $R_{m}$ and $R_{n}$ are the error for the approximate integration scheme.

Proof. By hypothesis, $\left(I+\mathcal{K}_{k}\right) \mu_{k}=(I+\mathcal{K}) \mu \operatorname{or}\left(I+\mathcal{K}_{k}\right)\left(\mu_{k}-\mu\right)=\left(\mathcal{K}-\mathcal{K}_{k}\right) \mu$ and so $\left(\mu_{k}-\mu\right)=\left(I+\mathcal{K}_{k}\right)^{-1}\left(\mathcal{K}-\mathcal{K}_{k}\right) \mu$. By $(4.5)$

$$
\left\|\mu_{k}-\mu\right\|_{H} \leq c\left\|\left(\mathcal{K}-\mathcal{K}_{k}\right) \mu\right\|_{H} .
$$

Furthermore, expanding the right hand side we have

$$
\begin{aligned}
& \left\|\left(\mathcal{K}_{k}-\mathcal{K}\right) \mu\right\|_{H}=\left\|\left(\mathcal{K}_{k}-\mathcal{K}\right)\left(\mu_{0}, \mu_{1}, A_{1}\right)\right\|_{H}= \\
& \operatorname{Max}\left\{\left\|\mathcal{K}_{m}^{00} \mu_{0}-\mathcal{K}^{00} \mu_{0}+\mathcal{K}_{n}^{01} \mu_{1}-\mathcal{K}^{01} \mu_{1}\right\|_{C\left(L_{0}\right)},\right. \\
& \left.\left\|\mathcal{K}_{m}^{10} \mu_{0}-\mathcal{K}^{10} \mu_{0}+\mathcal{K}_{n}^{11} \mu_{1}-\mathcal{K}^{11} \mu_{1}\right\|_{C\left(L_{1}\right)},\left|\mathcal{L}_{n} \mu_{1}-\int_{L_{1}} \mu_{1}(t) d t\right|\right\}
\end{aligned}
$$




$$
\begin{aligned}
& \leq \operatorname{Max}\left\{\left\|\left(\mathcal{K}_{m}^{00}-\mathcal{K}^{00}\right) \mu_{0}\right\|_{C\left(L_{0}\right)}+\left\|\left(\mathcal{K}_{n}^{01}-\mathcal{K}^{01}\right) \mu_{1}\right\|_{C\left(L_{0}\right)},\right. \\
& \left.\left\|\left(\mathcal{K}_{m}^{10}-\mathcal{K}^{10}\right) \mu_{0}\right\|_{C\left(L_{1}\right)}+\left\|\left(\mathcal{K}_{n}^{11}-\mathcal{K}^{11}\right) \mu_{1}\right\|_{C\left(L_{1}\right)},\left|\mathcal{L}_{n} \mu_{1}-\int_{L_{1}} \mu_{1}(t) d t\right|\right\} \\
& =\operatorname{Max}\left\{\begin{array}{c}
\operatorname{Max} \\
s \in L_{0}
\end{array}\left|\mathcal{K}_{m}^{00} \mu_{0}(s)-\mathcal{K}^{00} \mu_{0}(s)\right|+\underset{s \in L_{0}}{\operatorname{Max}}\left|\mathcal{K}_{n}^{01} \mu_{1}(s)-\mathcal{K}^{01} \mu_{1}(s)\right|,\right. \\
& \left.\underset{s \in L_{1}}{\operatorname{Max}}\left|\mathcal{K}_{n}^{10} \mu_{0}(s)-\mathcal{K}^{10} \mu_{0}(s)\right|, \underset{s \in L_{1}}{\operatorname{Max}}\left|\mathcal{K}_{n}^{11} \mu_{1}(s)-\mathcal{K}^{11} \mu_{1}(s)\right|,\left|\mathcal{L}_{n} \mu_{1}-\int_{L_{1}} \mu_{1}(t) d t\right|\right\} \\
& =\operatorname{Max}\left\{\begin{array}{c}
\operatorname{Max} \\
s \in L_{0}
\end{array}\left|R_{m}\left(\mathcal{K}^{00} \mu_{0}\right)\right|+\underset{s \in L_{0}}{\operatorname{Max}}\left|R_{n}\left(\mathcal{K}^{01} \mu_{1}\right)\right|+\right. \\
& \left.\underset{s \in L_{1}}{\operatorname{Max}}\left|R_{m}\left(\mathcal{K}^{10} \mu_{0}\right)\right|+\underset{s \in L_{1}}{\operatorname{Max}}\left|R_{n}\left(\mathcal{K}^{11} \mu_{1}\right)\right|,\left|R_{n}\left(\mu_{1}\right)\right|\right\} \text {. }
\end{aligned}
$$

From this result and the fact that we have chosen convergent numerical integration schemes, the method is convergent. Its rate of convergence will depend on the rate of convergence of the numerical integration schemes. We use the trapezoidal rule for quadrature since it achieves excellent convergence for smooth functions on periodic domains. The operator equation $\left(I+\mathcal{K}_{k}\right) \mu_{k}=f$, on $[0,2 \pi] \times[0,2 \pi] \times \mathbb{R}$ is equivalent to the $(m+n+1) \times(m+n+1)$ linear system

$$
\begin{aligned}
\mu_{k, 0}\left(t_{0, i}\right)+ & h_{m} \sum_{j=1}^{m} \mathcal{K}^{00}\left(t_{0, i}, t_{0, j}\right) \mu_{k, 0}\left(t_{0, j}\right)+h_{n} \sum_{j=1}^{n} \mathcal{K}^{01}\left(\left(t_{1, i}, t_{1, j}\right) \mu_{k, 1}\left(t_{1, j}\right)+\right. \\
& A_{1} l n\left|t_{0, i}-z_{1}\right|=f_{k, 0}\left(t_{0, i}\right), \quad i=1,2, \ldots, m \\
\mu_{k, 1}\left(t_{1, i}\right)+ & h_{m} \sum_{j=1}^{m} \mathcal{K}^{10}\left(t_{1, i}, t_{0, j}\right) \mu_{k, 0}\left(t_{0, j}\right)+h_{n} \sum_{j=1}^{n} \mathcal{K}^{11}\left(\left(t_{1, i}, t_{1, j}\right) \mu_{k, 1}\left(t_{1, j}\right)+\right. \\
& A_{1} \ln \left|t_{1, i}-z_{1}\right|=f_{k, 1}\left(t_{1, i}\right), \quad i=1,2, \ldots, n \\
& h_{n} \sum_{j=1}^{n} \mu_{k, 1}\left(t_{1, j}\right)=0 .
\end{aligned}
$$

\section{Iterative Methods}

The linear system (4.7) is dense and nonsymmetric. Solving this linear system using Gauss elimination will require $\mathcal{O}\left(N^{3}\right)$ operations. If the number of node points $N$ increases then an iterative algorithm will be necessary, one that requires a few iterations and is faster than Gauss elimination. Applied to a dense linear system, one iteration of an iterative methods usually requires order of $\mathcal{O}\left(N^{2}\right)$ operations.

We consider solving the linear system (4.7) using Krylov-subspace methods. Such methods produce approximation $x_{n}$ to the solution $x$ of the linear system $A x=b$ from the affine space $x_{0}+\mathcal{K}_{n}\left(r_{0}, A\right)$, where $x_{0}$ is the initial guess and the $n$th Krylov-subspace is $\mathcal{K}_{n}\left(r_{0}, A\right)=\operatorname{span}\left\{r_{0}, A r_{0}, \ldots, A^{n-1} r_{0}\right\}$ with $r_{0}=b-A x_{0}$, and with the property that the residual $r_{n}=b-A x_{n} \approx 0$ in some sense.

The iterative methods considered here are the GMRES (generalized minimal residual method) of Saad and Shultz [11], the Bi-CGSTAB (bi-conjugate gradient stabilized) of Van der Vorst [13], these two methods operate directly on the original system, and the CGN (the conjugate gradient method applied to the normal equation). The Conjugate Gradient (CG) due to Hestenes and Stiefel [5], only works 
for symmetric positive definite systems. The CGN algorithm refers to CG method applied to the normal equation $A^{T} A x=A^{T} b$ instead of $A x=b$. We remark that in the CGN algorithm the product $A^{T} A$ is never actually computed. Instead, whenever the product $A^{T} A x$ is needed by the CG algorithm, it carries out the product of two matrix-vector multiplications $A^{T}(A x)$. It is known that the convergence of the CGN method is determined by the singular values [10], the convergence of the GMRES is determined by the eigenvalues or pseudo-eigenvalues, and there is no convergence theory for the convergence of the Bi-CGSTAB method. These three method have been found to perform fundamentally different [10]. However, in applications of these methods to integral equations of second kind, we observed that the three methods have similar performance. This is shown in Table 2 and Figure 2. Convergence results for the CGN applied to Fredholm integral equations of second kind has been studied by Flores [3]. The dominant cost of the methods is in calculating the $N^{2}$ operations of the matrix-vector product. The CGN and the Bi-CGSTAB methods require two matrix-vector multiplications per iteration meanwhile the GMRES method only requires one matrix-vector multiplication per iteration. The storage requirements for the CGN and the Bi-CGSTAB methods is of order $\mathcal{O}\left(N^{2}\right)$ per iteration while the $k$ th iteration of GMRES has an storage requirement of order $\mathcal{O}\left(k N+N^{2}\right)$.

\section{Numerical Examples}

We solve the Dirichlet problem over a region bounded by two kinds of curves, the outer boundary is an ellipse and the inner boundary is an oval of Cassini, Figure 1.

In this example we look at the effect of the absolute error for the function $u(x, y)=y /\left(x^{2}+y^{2}\right)$, this function is also used as the true solution to obtain the error in the approximation. The points inside the region where the harmonic function is evaluated has been chosen in order to study the accuracy of the method in different parts of the region. Table 1 shows that the solution decreases its accuracy as the point where it is evaluated is closer to the boundary. Also, we have noted from Table 1 that the largest error is obtained where the point is close to the boundary. Table 2 shows that in order to to improve the accuracy at the point $(0,0.23)$ we need to increase the number of nodal points in both boundaries, and consequently the order of the linear system (4.7) needs to be increased. Table 2 and Figure 2 shows the numerical behaviour of the iterative methods CGN, GMRES and the BiCgSt. We noted that the number of iteration for all methods remain constant, this is known as mesh-independence principle, this behaviour which is due to the distribution of the eigenvalues of the compact integral operator has been analized in Flores [3]. Numerical experiments show that the speed of convergence at the point farther from the boundary behaves in accordance with the theoretical error formulas for the trapezoidal rule used in the integration. However, a glance at Table 1 shows that at the point closer to the boundary the speed of convergence is dramatically slower. To increase the accuracy we need to increase the number of nodal points. In Table 2 the columns label $C G N, G M R E S$ and BiCgSt represent the number of steps needed by the respective iterative method to achieve an approximating solution. The iterations were stopped when the norm of the residual $\left\|b-A_{n}\right\|<1.0^{-10}$. 
Table 1: Error as a function of closesness to the boundary

\begin{tabular}{ccc}
\hline$(x, y)$ & Error $(n, m=32)$ & Error $(m, m=64)$ \\
\hline$(0.0, .229)$ & $2.4884 D+00$ & $4.9262 D-01$ \\
$(0.4,0.4)$ & $4.7207 D+00$ & $2.1179 D+00$ \\
$(0.6,0.6)$ & $7.5835 D-02$ & $5.7600 D-03$ \\
$(2.0,1.5)$ & $2.4319 D-05$ & $2.0363 D-07$ \\
$(2.12,1.76)$ & $7.8319 D-02$ & $1.9035 D-04$ \\
\hline \multicolumn{3}{c}{ See Figure: 1}
\end{tabular}

Table 2: Convergence of the iterative methods

\begin{tabular}{rrcccc}
\hline$m, n$ & order & CGN & GMRES & BiCgSt & Error \\
\hline 8 & 17 & 5 & 4 & 4 & $1.6890 D+01$ \\
16 & 33 & 11 & 7 & 6 & $6.8326 D+00$ \\
32 & 65 & 11 & 8 & 7 & $2.3013 D+00$ \\
64 & 129 & 11 & 8 & 8 & $4.7691 D-01$ \\
128 & 257 & 11 & 8 & 7 & $3.0519 D-02$ \\
256 & 513 & 12 & 8 & 7 & $1.4198 D-04$ \\
512 & 1025 & 12 & 8 & 7 & $3.1024 D-09$ \\
1024 & 2049 & 12 & 8 & 8 & $1.3722 D-12$ \\
\hline \multicolumn{5}{c}{ Iterative Methods at the point $(0.0,0.23)$}
\end{tabular}

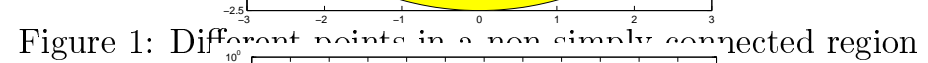
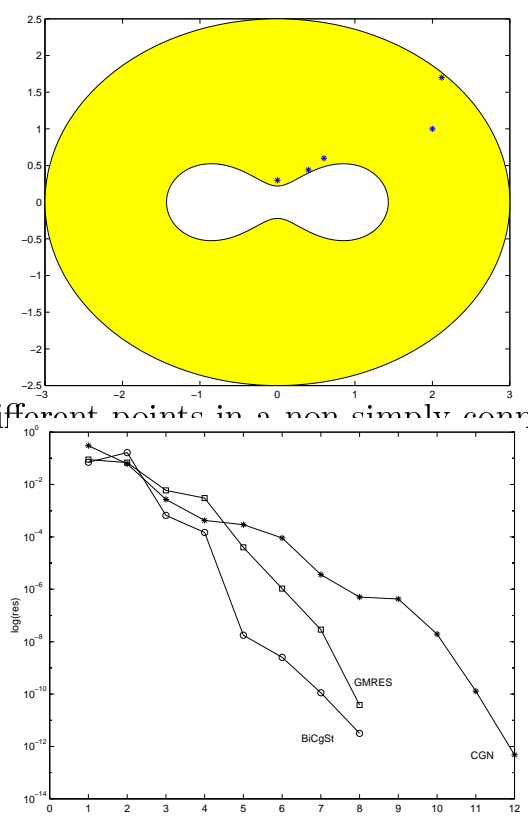

Figure 2: Rate of convergence for the iterative methods 


\section{References}

[1] P.M. Anselone, "Collectively Compact Operator Approximation Theory and Applications to Integral Equations", Prentice-Hall, Inc., 1971.

[2] K.E. Atkinson, "The Numerical Solution of Integral Equations of the Second Kind", Cambridge University Press, 1997.

[3] J.D. Flores, The conjugate gradient method for solving Fredholm integral equations of second kind, Intern. J. Comp. Math. 48 (1993), 77-94.

[4] N. Günter, "Potential Theory", Ungar Publishing, New York, 1967.

[5] M. Hestenes and E. Stiefel, Methods of conjugate gradients for solving linear systems, J. Res. Nat. Bur. Stand. 49 (1952), 409-439.

[6] O.D. Kellogg, "Fundation of Potential Theory", reprinted by Dover Publications, New York, 1929.

[7] W. McLean, "Boundary Integral Method for Laplace Equation", Ph.D. Thesis, Australian National University, Camberra, 1985.

[8] S.G. Mikhlin, "Integral Equations", 2nd ed. trans. by A.H. Armstrong, Pergamon Press, London, 1966.

[9] N.I. Muskhelishvili, "Singular Integral Equations", Noordhoff, 1946.

[10] N. Nachtigal, S. Reddy, and L. Trefethen, How fast are nonsymmetric matrix iterations?, SIAM J. Matrix Anal. Appl. 13 (1992), 778-795.

[11] Y. Saad and M. Shultz, GMRES: A generalized minimal residual algorithm for solving nonsymmetric linear systems, SIAM J. Sci. Stat. Comput. 7 (1986), 856-869.

[12] J. Saavedra, "Numerical Solution for Laplace's Equation on a Non-Simply Connected Regions", Ph.D. Thesis, The University of Iowa, May 1988.

[13] H. Van der Vorst, BI-CGSTAB: A fast and smoothly converging variant of BI-CG for the solution of nonsymmetric linear systems, SIAM J. Sci. Stat. Comput. 13 (1992), 631-644. 